\title{
Sensitive Quantification of Nicotine in Bronchoalveolar Lavage Fluid by Acetone Precipitation Combined with Isotope-Dilution Liquid Chromatography-Tandem Mass Spectrometry
}

Baoyun Xia, ${ }^{1 *}$ Benjamin C Blount, ${ }^{1}$ and Lanqing Wang ${ }^{1 *}$

${ }^{1}$ Tobacco and Volatiles Branch, Division of Laboratory Sciences, National Center for Environmental Health, U.S. Centers for Disease Control and Prevention (CDC), 4770 Buford Highway Mail Stop S103-3, Atlanta, GA 30341

*Corresponding Authors:

Tobacco and Volatiles Branch, Division of Laboratory Sciences, National Center for Environmental Health, U.S. Centers for Disease Control and Prevention (CDC), 4770 Buford Highway Mail Stop S103-3, Atlanta, GA 30341

Baoyun Xia, phone: 770-488-0148, e-mail: vvq2@cdc.gov

Tobacco and Volatiles Branch, Division of Laboratory Sciences, National Center for Environmental Health, U.S. Centers for Disease Control and Prevention (CDC), 4770 Buford Highway Mail Stop S103-3, Atlanta, GA 30341

Lanqing Wang, phone: 770-488-7914, e-mail: $\underline{\text { ffw3@cdc.gov }}$

Contents of Supplemental Files:

Table S1. Factors which Effect the Nicotine Sensitivity and Baseline Noise.

Table S2. Standard Curve Prepared in Water.

Table S3. Standard Curve with Saline Matrix and Process as Unknowns.

Table S4. Standard Curve with BAL Fluid Matrix and Process as Unknowns.

Figure S1 Comparison of Nicotine LC-MS/MS Profile of A) Before system clean up and B) After system clean up. 
Table S1. Factors which Effect the Nicotine Sensitivity and Baseline Noise.

\begin{tabular}{|c|c|c|}
\hline $\begin{array}{l}\text { Factors effect nicotine } \\
\text { sensitivity and baseline noise }\end{array}$ & Different conditions & Optimized and Selected \\
\hline Water & $\begin{array}{l}\text { Water (J.T. Baker, Cat\# 4218-03), Water (Tedia Company, Inc., Cat. } \\
\text { \#WS2211001), Water (Fisher Scientific, Cat\#w6-4) }\end{array}$ & $\begin{array}{l}\text { Water (Tedia Company, } \\
\text { Inc., Cat. \#WS2211001) }\end{array}$ \\
\hline Acetonitrile & $\begin{array}{l}\text { Acetonitrile (Fisher Scientific, Cat \#A955-4), Acetonitrile (Honeywell, Cat } \\
\text { \#AH015-4), }\end{array}$ & $\begin{array}{l}\text { Acetonitrile (Honeywell. } \\
\text { Cat \#AH015-4) }\end{array}$ \\
\hline Mobile phase A & $\begin{array}{l}\text { Ammonium acetate ( } \mathrm{pH} 4.5) \text {, ammonium acetate }(\mathrm{pH} 6.2) \text {, ammonium } \\
\text { acetate }(\mathrm{pH} \text { 10.5), Ammonium hydroxide } 0.05 \%(\mathrm{pH} 10.5)\end{array}$ & $\begin{array}{l}\text { Ammonium hydroxide } \\
0.05 \%(\mathrm{pH} 10.5)\end{array}$ \\
\hline $\begin{array}{l}\text { Different brands ammonium } \\
\text { hydroxides }\end{array}$ & $\begin{array}{l}\text { Ammonium hydroxide (Sigma Aldrich, Cat\# 44273-10x } 1 \mathrm{ML}-\mathrm{F} \text { ), } \\
\text { Ammonium hydroxide (Fisher Scientific, Cat. \#A669S-500) }\end{array}$ & $\begin{array}{l}\text { Ammonium hydroxide } \\
\text { (Fisher Scientific, Cat. } \\
\text { \#A669S-500) }\end{array}$ \\
\hline HPLC columns & $\begin{array}{l}\text { Phenomenex, Gemini NX C18, 3.0um,2.1×150mm, Agilent, Eclipse plus } \\
\mathrm{C} 18,3.5 \mathrm{um}, 2.1 \times 100 \mathrm{~mm} \text {, Agilent Poroshell, HPH-C18, } 2.1 \times 100 \mathrm{~mm} 2.7 \\
\mu \mathrm{m} ;\end{array}$ & $\begin{array}{l}\text { Agilent Poroshell, HPH-C18, } \\
2.1 \times 100 \mathrm{~mm} 2.7 \mu \mathrm{m}\end{array}$ \\
\hline Temperature of column & $25^{\circ} \mathrm{C}, 40^{\circ} \mathrm{C}, 50^{\circ} \mathrm{C}, 55^{\circ} \mathrm{C}$ & $55^{\circ} \mathrm{C}$ \\
\hline $\begin{array}{l}\text { Different percentage mobile } \\
\text { phase B (acetonitrile) in time } \\
\text { gradient }\end{array}$ & $\begin{array}{l}3 \% \text { acetonitrile, } 5 \% \text { acetonitrile, } 10 \% \text { acetonitrile, } 20 \% \text { acetonitrile, } 23 \% \\
\text { acetonitrile, } 30 \% \text { acetonitrile }\end{array}$ & $\begin{array}{l}23 \% \text { acetonitrile (mobile } \\
\text { phase } B \text { ) }\end{array}$ \\
\hline
\end{tabular}

Table S2. Standard Curve Prepared in Water

\begin{tabular}{cccccc}
\hline $\begin{array}{c}\text { Stock } \\
\text { solution in } \\
\text { water }(\mathrm{ng} / \mathrm{mL})\end{array}$ & $\begin{array}{c}\text { Stock } \\
\text { solution } \\
\text { volume }(\mathrm{mL})\end{array}$ & ISTD $^{*}(\mathrm{~mL})$ & Water $(\mathrm{mL})$ & $\begin{array}{c}\text { Total } 100 \mathrm{~mL} \\
\text { flask } \\
\text { volume }(\mathrm{mL})\end{array}$ & $\begin{array}{c}\text { Final } \\
\text { concentration } \\
(\mathrm{ng} / \mathrm{mL})\end{array}$ \\
\hline 0.000 & 10.0 & 10.0 & add water to scale & 100.0 & 0.000 \\
0.050 & 10.0 & 10.0 & add water to scale & 100.0 & 0.005 \\
0.125 & 10.0 & 10.0 & add water to scale & 100.0 & 0.013 \\
0.250 & 10.0 & 10.0 & add water to scale & 100.0 & 0.025 \\
0.500 & 10.0 & 10.0 & add water to scale & 100.0 & 0.050 \\
1.25 & 10.0 & 10.0 & add water to scale & 100.0 & 0.125 \\
2.50 & 10.0 & 10.0 & add water to scale & 100.0 & 0.250 \\
5.00 & 10.0 & 10.0 & add water to scale & 100.0 & 0.500 \\
12.5 & 10.0 & 10.0 & add water to scale & 100.0 & 1.25 \\
25.0 & 10.0 & 10.0 & add water to scale & 100.0 & 2.50 \\
37.5 & 10.0 & 10.0 & add water to scale & 100.0 & 3.75 \\
50.0 & 10.0 & 10.0 & add water to scale & 100.0 & 5.00 \\
\hline
\end{tabular}

*ISTD: Nicotine_ ${ }^{13} \mathrm{CD}_{3} 12.5 \mathrm{ng} / \mathrm{mL}$ in water. 
Table S3. Standard Curve with Saline Matrix and Process as Unknowns

\begin{tabular}{ccccccc}
\hline $\begin{array}{c}\text { Stock solution in } \\
\text { saline }(\mathrm{ng} / \mathrm{mL})\end{array}$ & $\begin{array}{c}\text { Stock } \\
\text { solution } \\
\text { volume }(\mu \mathrm{L})\end{array}$ & Water $(\mu \mathrm{L})$ & ISTD $(\mu \mathrm{L})$ & Acetone $(\mu \mathrm{L})$ & $\begin{array}{c}\text { Total } \\
\text { volume }(\mu \mathrm{L})\end{array}$ & $\begin{array}{c}\text { Final } \\
\text { concentration } \\
(\mathrm{ng} / \mathrm{mL})\end{array}$ \\
\hline 0.000 & 40 & 40 & 40 & 280 & 400 & 0.000 \\
0.050 & 40 & 40 & 40 & 280 & 400 & 0.005 \\
0.125 & 40 & 40 & 40 & 280 & 400 & 0.013 \\
0.250 & 40 & 40 & 40 & 280 & 400 & 0.025 \\
0.500 & 40 & 40 & 40 & 280 & 400 & 0.050 \\
1.25 & 40 & 40 & 40 & 280 & 400 & 0.125 \\
2.50 & 40 & 40 & 40 & 280 & 400 & 0.250 \\
5.00 & 40 & 40 & 40 & 280 & 400 & 0.500 \\
12.5 & 40 & 40 & 40 & 280 & 400 & 1.25 \\
25.0 & 40 & 40 & 40 & 280 & 400 & 2.50 \\
37.5 & 40 & 40 & 40 & 280 & 400 & 3.75 \\
50.0 & 40 & 40 & 40 & 280 & 400 & 5.00 \\
\hline
\end{tabular}

*ISTD: Nicotine_- ${ }^{13} \mathrm{CD}_{3} 12.5 \mathrm{ng} / \mathrm{mL}$ in water.

Table S4. Standard Curve with BAL Fluid Matrix and Process as Unknowns

\begin{tabular}{ccccccc}
\hline $\begin{array}{c}\text { Stock solution in } \\
\text { BAL fluid }(\mathrm{ng} / \mathrm{mL})\end{array}$ & $\begin{array}{c}\text { Stock } \\
\text { solution } \\
\text { volume }(\mu \mathrm{L})\end{array}$ & Water $(\mu \mathrm{L})$ & ISTD $(\mu \mathrm{L})$ & Acetone $(\mu \mathrm{L})$ & $\begin{array}{c}\text { Total } \\
\text { volume }(\mu \mathrm{L})\end{array}$ & $\begin{array}{c}\text { Final } \\
\text { concentration } \\
(\mathrm{ng} / \mathrm{mL})\end{array}$ \\
\hline 0.000 & 40 & 40 & 40 & 280 & 400 & 0.000 \\
0.050 & 40 & 40 & 40 & 280 & 400 & 0.005 \\
0.125 & 40 & 40 & 40 & 280 & 400 & 0.013 \\
0.250 & 40 & 40 & 40 & 280 & 400 & 0.025 \\
0.500 & 40 & 40 & 40 & 280 & 400 & 0.050 \\
1.25 & 40 & 40 & 40 & 280 & 400 & 0.125 \\
2.50 & 40 & 40 & 40 & 280 & 400 & 0.250 \\
5.00 & 40 & 40 & 40 & 280 & 400 & 0.500 \\
12.5 & 40 & 40 & 40 & 280 & 400 & 1.25 \\
25.0 & 40 & 40 & 40 & 280 & 400 & 2.50 \\
37.5 & 40 & 40 & 40 & 280 & 400 & 3.75 \\
50.0 & 40 & 40 & 40 & 280 & 400 & 5.00 \\
\hline
\end{tabular}

*ISTD: Nicotine_ ${ }^{13} \mathrm{CD}_{3} 12.5 \mathrm{ng} / \mathrm{mL}$ in water. 

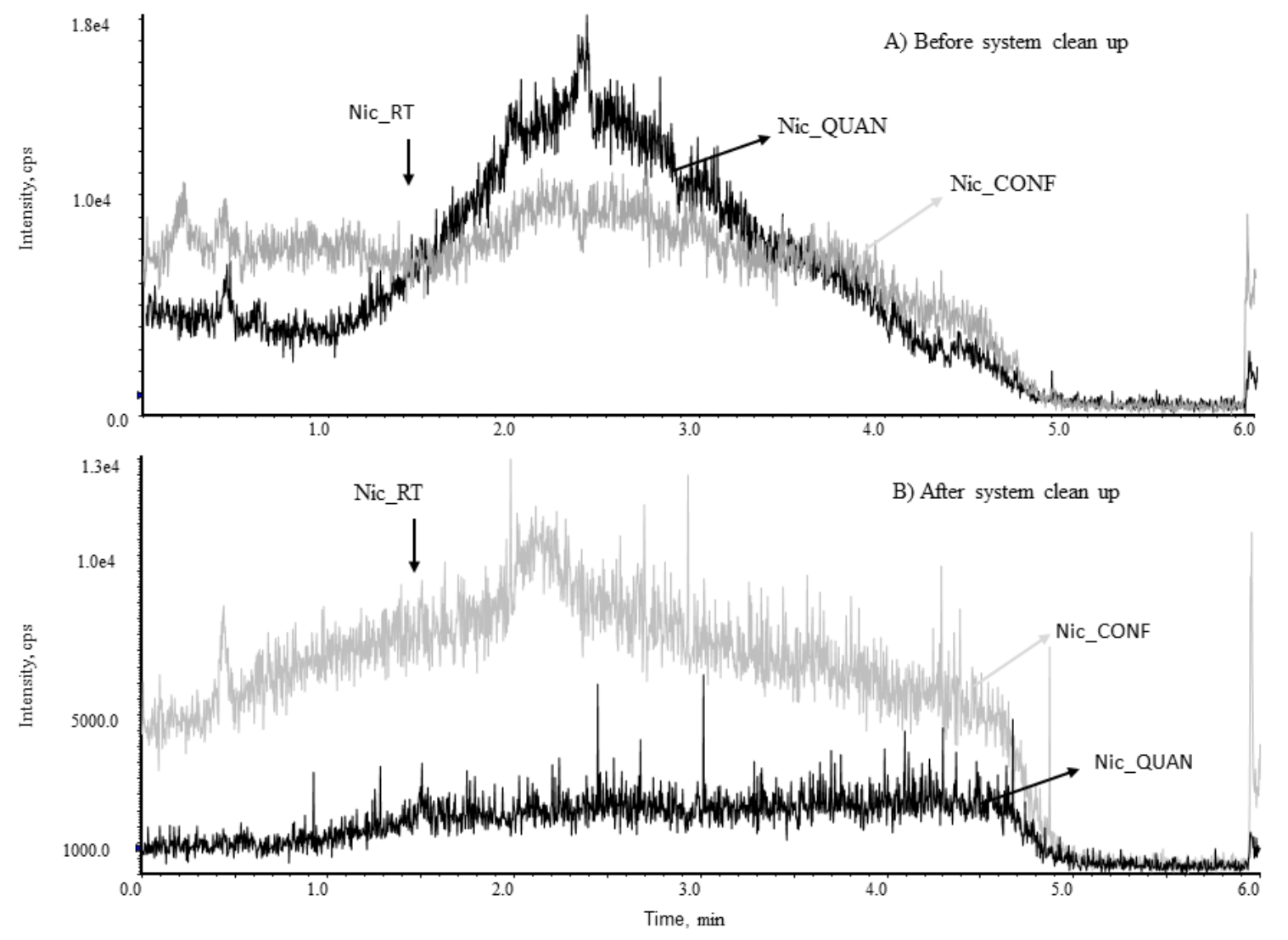

Figure S1 Comparison of Nicotine LC-MS/MS Profile of A) Before system clean up and B) After system clean up. Nic_RT: nicotine peak retention time; NiC_QUAN: nicotine quantitation transition (163.1/130.0); Nic_CONF: nicotine confirmation transition (163.1/117.0). 